WORISHOP ON POLARIZED PROTON BEAMS

ANN ARBOR, MICHIGAN, OCTOBER 18-27, 1977

ACCELERATION AND STORAGE OF POLARIZED BBAMS

E. D. Courant *

BNL and SUNY, Stony Brook

\title{
INTRODUCTION
}

\section{Review of Theory}

The polarization of a beam in a circular accelerator or storage ring is affected by depolarizing resonances. The theory of these resonances is well known; however, I shall formulate it here in such a way as to facilitate quantitative estimates, in terms of the detailed characteristics of a particular accelerator.

The Frolssart-stora (FS) equation is:

$$
\frac{d \vec{S}}{d t}=\frac{e}{\gamma m c} \vec{S} \times\left[(1+y G) \vec{B}_{\perp}+(1+G) \vec{B}_{\|}\right]
$$

where $G=(8-2) / 2$ In the gyromagnetic anomaly, and $\vec{B}_{\perp}$ and $\vec{B}_{\|}$are the parts of the magnetic field perpendicular and parallel to the direction of motion.

It is useful to remite the FS equation in terms of the motion of the particle in the field of the accelerator. We assume the accelerator or storage ring has a reference orbit lying in the horfzontal plane (not zecessarily the equilibrium orbit of a particle - field errors may shift the particle orbit from the reference orbit). Define the following local coordinate system. 2

*Work performed under the auspices of the U.S. Department of Energy. 1. M. Froissart and R. Stora, Nucl. Instrum. $I, 297$ (1960).

2. See, for example. E.D. Courant and H.S. Snyder, Ann. Phys. 3, 1 (1958). 
$s$ = distance along reference orbit

$P(s)=$ radius of curvature

$\vec{b} \quad=$ unit vector along reference orbit

$\vec{a} \quad=$ unit vector in orbit plane transverse to orbit

$\vec{c}=\vec{a} \times \vec{b}=$ vertical unit vector

The equation of motion of the particle:

$$
\frac{d \vec{v}}{d t}=\frac{\theta}{\gamma m c} \vec{v} \times \vec{B}
$$

then enables us to trite $\vec{B}_{\perp}$ and $\vec{B}_{\|}$in terms of the radius of curvature and the coordinates of motion of the particle (Including the deviations from the reference orbit due to field errors as well as betatron oscillations):

$$
\frac{d \vec{S}}{d s}=\vec{S} \times \vec{F}
$$

with

$$
\vec{F}=\left[(1+G)\left(\frac{z}{p}\right)^{\prime}-(1+\gamma G) \frac{z^{\prime}}{\rho}\right] \vec{b}+(1+\gamma G)\left[z^{\prime \prime} \vec{a}+\frac{\vec{c}}{\rho}\right]
$$

where $z$ is the vertical excursion of the particle and the prime : denotes differentiation by $s$.

Now describe the spin vector $\vec{s}$ in terms of 1 ts components $s_{1}$, $s_{2}, s_{3}$, in. the $\vec{a}, \vec{b}, \vec{c}$ directions; introduce as the new independent variable the turning angle $\theta=\sqrt{d s} / \rho$ and formulate the spin motion in terms of 2-component spinors rather than 3-component vectors (as first done by Hamilton a century ago). With the wave function $\downarrow$, we have:

$$
s_{i}=\psi^{+} \sigma_{i} \psi 1, i=1,2,3
$$

where $\sigma_{1}$ are the Paull spin maticices, and we find:

where

$$
\frac{d y}{d \theta}=\cdot \frac{1}{2}\left(\begin{array}{cc}
-x & \zeta \\
\zeta^{*}+x
\end{array}\right)
$$

and

$$
x=\gamma G
$$

$$
\begin{aligned}
S(s) & =-p\left(F_{a}-1 F_{b}\right) \\
& =-(1+x)\left(p z^{\prime \prime}+i z^{\prime}\right)+i p(1+G)\left(\frac{z}{\rho}\right)^{\prime}
\end{aligned}
$$


For a particle on the reference orbit, $\zeta=0$, and $\sigma_{3}$ is constant, while $\sigma_{1}$ and $\sigma_{2}$ precess with frequency $x_{\text {. But }}{ }^{\text {if }} \zeta$ possesses a component oseillating as $e^{-i \chi \theta}$, resonance will occur.

The irequencies contained in $S$ are determined by the behavior of $z(\theta)$. Accelerator orbit theory tells us that, in general

$$
z(\theta)=z_{e}(\theta)+\sqrt{e \beta(\theta)}\left[e^{-i[v \theta-x(\theta)]}+c_{. c}\right]
$$

where: $Z_{\hat{e}}(\theta)$ is the closed orbit whose deviation from zero is produced by field errors, and the second term is the betatron oscillation; here $v$ is the "tune" or oscillation frequency, $\beta(\theta)$ and $x(\theta)$ are certain perlodic functions of $\theta$ having the azimuthal periodicity of the accelerator structure. The radius of curvature $p$ has this same periodicity. $e$ is the emittance.

Therefore, components with frequency $x$, exist in $\zeta$ if we have:

$$
x=k P \pm v
$$

for structure resonances excited by betatron oscillations, where $P$ is the number of identical periods in the accelerator structure and $k$ is any integer. Imperfection resonances arise at all integral values of $\mu$, since the closed orbit contains all hamonics; the most prominent ones are again likely to be the ones near the struc zure resonances.

\section{EFFECT OF RESONANCE}

\section{a. Steady State}

If the fields are time-independent, we suppose that we are a distance $\delta$ from a resonant value of $u$, and that $\zeta$ contains $a$ resonant component. Then (4) betomes:

$$
\frac{d v}{d \theta}=-\frac{1}{2}\left[\begin{array}{cc}
x_{0}+8 & \varepsilon_{0}^{-i x_{0} \theta} \\
\epsilon e^{1 x_{0} \theta} & -\left(x_{c}+8\right)
\end{array}\right]
$$

This is easily solved; in general, the polarization,

$$
P=S_{3}=t^{+} \sigma_{3}
$$

oscillates rapidly around a mean value. The maximum time-average or steady-state polarization can be seen to be: 


$$
\begin{gathered}
-4- \\
P_{\max }=\frac{8}{\sqrt{6 \epsilon^{t}+8^{2}}}
\end{gathered}
$$

Therefore, $|\epsilon|$ may be called the width of the resonance.

b. Passage through Resonance

Now if one passes through a resonance by changing 6 with time from negative to positive, this equilibrium spin will change sign. This is just the phenomenon of "fast adiabatic passage" familiar in the field of magnetic resonance in solid-state phystcs (the term "fast" refers to relaxation times which are irrelevant in our context).

In general, passage through resonance is analyzed by putting $6=\alpha \theta$ in (9), where:

$$
\alpha=\frac{d x}{d \theta}=\frac{1}{2 \pi} \quad(G \Delta y \pm \Delta v)
$$

where $\Delta y$ is the energy gain per turn and, for betatron resonance, . I $\Delta \nu$ is the tune shift per turn due to pulsed quadrupoles such as those used at Argonne. [For imperfection resonances the $\Delta v$ term in (11) has to be omfted.]

The solution, first given by Froissart and Stora, ${ }^{1}$ is obtained by transforming (9) to a confluent hypergeometric equation. It is found that, with initial polarization $P_{1}$, the polarization after passage through resonance is:

$$
P_{2}=P_{1}\left(2 e^{-\pi|\epsilon|^{2} / 2 \alpha}-1\right)
$$

therefore, polarization is present to the extent of $99 \%$ if,

$$
x=\frac{\pi}{2} \frac{|\epsilon|^{2}}{\alpha}<0.005, \text { or }|\epsilon|<0.03 \sqrt{\Delta \gamma \pm \Delta \nu / G}
$$

(for protons with $G=1.79$ )

Spiñ-flip is 99\% complete, $1 . \ddot{e} .$, polarization is present with change of sign, if,

$$
\begin{aligned}
& |x|>\ln 200=5.30, \\
& |\epsilon|>0.980 \sqrt{\Delta y \pm \Delta v / G}
\end{aligned}
$$


In practice, it is found at Argonne that complete spin-flip is difficult to obtain, probably because synchrotron oscillations en: sure that not all particles have the same $\Delta Y$, and also because some particles have a small emittance and, therefore, a small value of $\varepsilon$.

\section{EVALUATION OF RESONANCE WIDTH}

The strength $\&$ of a. Iesonance is obtained by finding the component oscillating as $e^{-i \mu \theta}$ in $\zeta$ as given by (6):

$$
=c=\frac{1}{2 \pi} \cdot \int_{0}^{2 \pi} S e^{i x \theta} d \theta
$$

In a lattice raade up of bending magnets (with or without gradiants and edge focusing) and quadrupoles, one can evaluate (13) analgtically, using ( 7 ) and the equations of motion satisfled by $z(\theta)$. It is found that, for the structure resonance $u=\gamma_{G}=k \pm v$, the contributioh of a magnet with curvature $1 / f$, gradient $R B p$ (R positive for vertical focusing), and entrance and exit wedge angles $S_{1}$ and $S_{2}$ is:

$$
\begin{aligned}
\epsilon= & \frac{\sqrt{e}}{4 \pi}\left\{\left[c+\frac{(1+x)}{\rho}\left(\zeta_{1}+1\right)\right] \beta_{1}^{\frac{1}{2}} e^{i x_{1}}\right. \\
& +\left[c^{*}+\frac{(1+x)}{\rho}\left(\zeta_{2}-1\right)\right] \beta_{2}^{\frac{1}{2}} e^{i \gamma_{2}}
\end{aligned}
$$

where,

$$
c=\frac{\left[(1+x) R \rho^{2}-x(x-G)\right]}{x^{2}-R \rho^{2}}\left[\frac{\sqrt{R}\left(\cos l \sqrt{R}-e^{1 x \theta}\right)}{\sin \sqrt{k}}+\frac{i x}{\rho}\right]
$$

where $\ell=$ Iength of magnet

$$
\theta=\ell / p=\text { turning angle of magnet. }
$$

The amplitude function $\beta$ and the phase function $X=v_{\theta}-\phi(\psi=$ betatron oscillation phase) may be obtained at the beginning and end of each magnet using computer programs such as SYNCH or AGs.

In the smooth approximation for a perfect machine, only the resonance $x=v$ comes into play; in that case $R p^{2}=v, \beta=R / v$, 
$x=0, \theta=2 \pi$ and (15) becomes, for the complete ring,

$$
c=\frac{2}{2} \sqrt{\frac{2}{R}} x^{\frac{2}{2}}\left(x^{2}+G\right)
$$

In the case of imperfection resonances (at integral values of $x$ ) one obtains, for the contribution of a magnet,

$$
\begin{aligned}
\epsilon= & \frac{1}{2 \pi}\left\{\left[c+\frac{(1+x)}{p}\left(\zeta_{1}+1\right)\right] z_{1}\right. \\
& \left.+\left[c^{*}+\frac{(1+x)}{p}\left(\zeta_{2}-1\right)\right] z_{2}\right\}
\end{aligned}
$$

where $z_{1}$ ind $z_{2}$ are the displacenents of the closed orbit at the entrance and exit of the magnet.

\section{POSSIBILITIES ÓF ACCELERATING POLARIZED PARTICLES}

The computer program SYNCH or AGS (or equivalent) can evaluate the parameter needed for (14) and (17) for each magnet, and another program DEPOL has been written to evaluate (14) and (17) using the output of SYNCH. For the Brcokhaven AGS, with magnets randomiy misaligned by \pm 0.1 man with a beam emittance of $(10 \pi / Y) \times 10^{-6}$ meter-radians, the results are shown in Fig. 1. Here the two horizontal dashed lines correspond to a depolarization of less than : 1\% per resonance (lower line) and $99 \%$ spin reversal (upper line.)

It is seen that below about $20 \mathrm{GeV}$, there are six intrinsic resonances and about efght imperfection resonances, strong enough to depolarize the beam by more than $1 \%$.

Comparable resonances have been overcome at the Argonne $2 G S$ by fast pulsing of the tune and careful adjustment of magnet orbit errors. It might be feasible to do the same at the AGS (OI CERN PS or similar machines) at energies up to about 20-25 GeV.

A complementary method may be to enhance resonances - or slow passage through them - until they become strong enough for complete spin-flip. Argonne's experience shows that this may be difficult, probably because synchrotron oscillations prevent all particles from passing through a resonance slowly.

It thus appears possible - but not easy - to maintain proton 
polarization in the AGS up to about $20 \mathrm{GeV}$.

An alternative approach is to consider deuterons. The deuteron anomalous moment $G$, Is only -0.14 and its mass is twice that of the proton; therefore, the resonances are spaced 25 times as far apart in energy as for protons. Thus, only two imperfection resonances, and no intrinsic resonances, exist in the AGS below $30 \mathrm{GeV}$.

Polarization in storage rings is feasiile if the polarization in the steady-st ate does not decrease too fast. The simple theory above does not predict ang effect of this sort, however, scattering, beam-beam interactions, field and energy fluctuations, etc. might conceivably produce slow depolarization. (Experiments at ANL, so far, have shown no evidence of such effects, but have been Iimited to short storage times.)

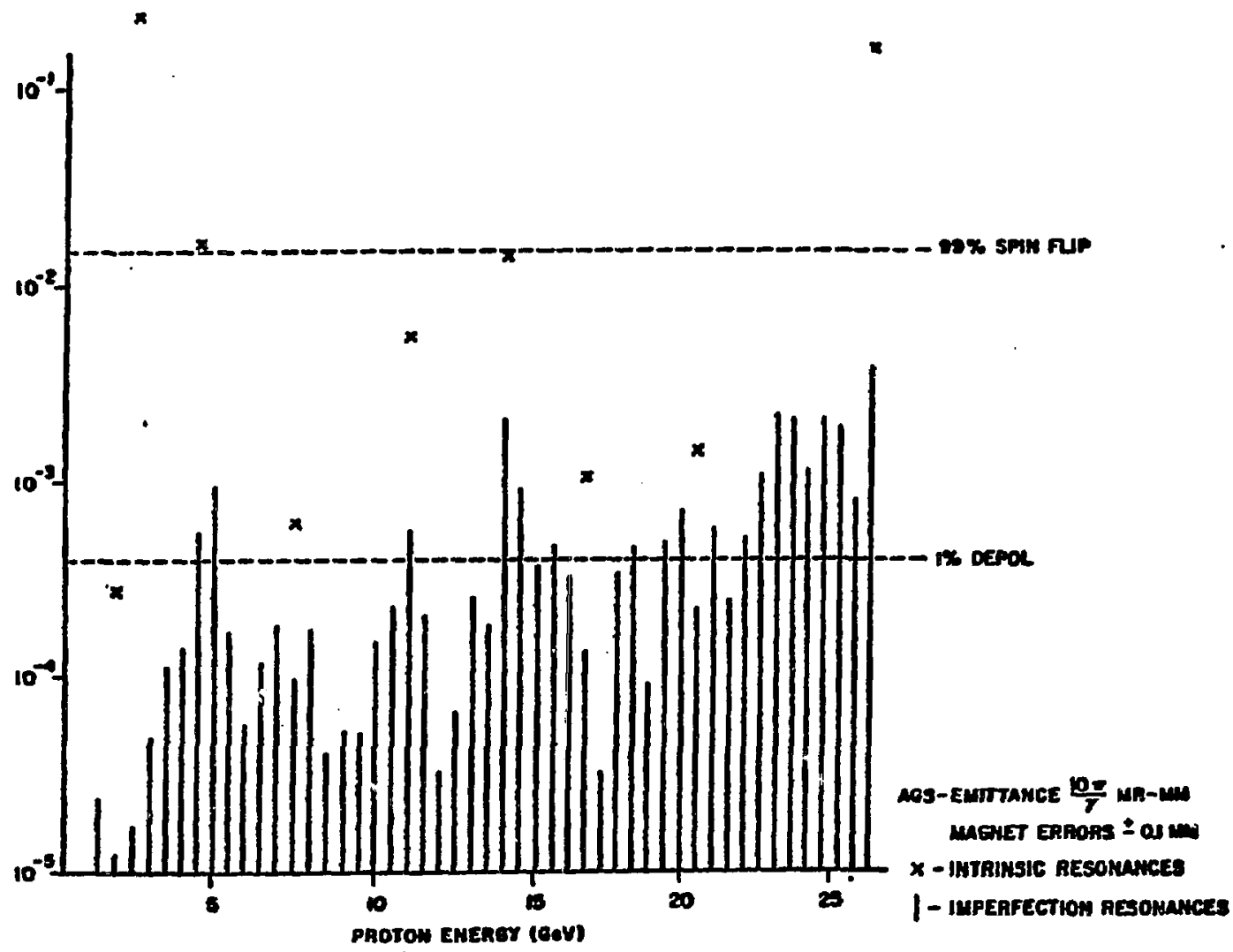

F18. 1. 